\title{
Métropolisation et santé à Orléans : quand l'institution métropolitaine ouvre de nouveaux champs d'action
}

Pierre Allorant, Sylvain Dournel et Fouad Eddazi

\section{OpenEdition}

Édition électronique

URL : https://journals.openedition.org/rfst/1502

DOI : $10.4000 /$ rfst.1502

ISSN : 2492-3672

Traduction(s) :

Metropolisation and health in Orléans : when the metropolitan institution allows new territorial actions

- URL : https://journals.openedition.org/rfst/1547 [en]

Éditeur

Espaces et SOciétés (UMR 6590)

Référence électronique

Pierre Allorant, Sylvain Dournel et Fouad Eddazi, « Métropolisation et santé à Orléans : quand

l'institution métropolitaine ouvre de nouveaux champs d'action », Revue francophone sur la santé et les territoires [En ligne], Décentralisation et territorialisation sanitaire, mis en ligne le 19 janvier 2022, consulté le 14 février 2022. URL : http://journals.openedition.org/rfst/1502 ; DOI : https://doi.org/ 10.4000/rfst.1502

Ce document a été généré automatiquement le 14 février 2022.

La Revue francophone sur la santé et les territoires est mise à disposition selon les termes de la Licence Creative Commons Attribution - Pas d'Utilisation Commerciale - Partage dans les Mêmes Conditions 4.0 International. 


\title{
Métropolisation et santé à Orléans : quand l'institution métropolitaine ouvre de nouveaux champs d'action
}

\author{
Pierre Allorant, Sylvain Dournel et Fouad Eddazi
}

\section{Contexte de la recherche}

1 Le présent article expose les premiers résultats d'une recherche-action conduite entre 2019 et 2022 à l'échelle de l'aire urbaine d'Orléans. Portant sur les liens entre santé et territoires, cette recherche-action s'inscrit dans le cadre d'un vaste programme national axé sur 15 métropoles et leurs territoires voisins, porté par la Plate-forme d'observation des projets et stratégies urbaines (POPSU). Les travaux menés dans l'Orléanais rassemblent une dizaine de scientifiques de l'Université d'Orléans, dont un ingénieur de recherche et un doctorant, émanant de l'aménagement, du droit public, de la géographie, de l'histoire du droit, de la science politique, des sciences de la gestion et des STAPS. Cette recherche-action est à la fois soutenue par Orléans Métropole et l'État, via le programme POPSU, ainsi que par 2 PETR et la région Centre-Val de Loire, via le dispositif « A VOS ID ».

2 Les observations faites dans cet article proviennent de la confrontation de trois sources de données :

- la mise en exergue des principaux enseignements d'un séminaire et d'une journée d'étude, tous deux tenus à la fin de l'année 2019 ;

- l'exploitation de données d'organismes ressources sur les liens entre santé et territoires (ARS, TOPOS, CESER, INSEE, Orléans Métropole, etc.) ;

- la valorisation des réflexions de quelques grands témoins de l'Orléanais (chefs de service santé et directeurs généraux de service, chercheurs, élus intercommunaux et régionaux, professeurs de médecine et membres du conseil de développement métropolitain) issues de deux phases d'enquêtes par questionnaire, en juillet 2019 et octobre 2021. 


\section{Les motifs de l'intervention métropolitaine}

3 Les liens entre métropolisation et santé interrogent (Jomier \& Surel, 2017 ; Allorant et al., 2021). D'emblée, la métropolisation, cette expression urbaine de la mondialisation (Veltz, 1997), est mise en cause pour sa responsabilité dans la diffusion foudroyante de la pandémie de COVID-19. Plus généralement, les métropoles ont l'image d'attractives entités de plusieurs millions d'habitants particulièrement pourvues en équipements structurants, sièges sociaux de grandes entreprises, services supérieurs et institutions de renom, mais font simultanément l'objet de contestations sociales autour de la dégradation de la qualité de l'air, de la consommation des ressources naturelles, de l'artificialisation des sols, de l'exposition des personnes et des biens aux risques environnementaux et technologiques (Dab \& Roussel, 2001 ; Larrouy-Castera \& Ourliac, 2004 ; Salomon-Cavin, 2005 ; Pigeon, 2007, 2012)... Cette réalité urbaine parait alors éloignée de la définition de la santé faite par l'organisation mondiale de la santé (OMS) : « un état de complet bien-être physique, mental et social, et ne consiste pas seulement en une absence de maladie ou d'infirmité ».

4 Pour autant, s'agit-il bien de ces questions quand on évoque les liens de métropolisation et santé à Orléans ? Certes, cette agglomération de 280000 habitants, à la tête d'une aire urbaine de plus de 400000 habitants, tire parti sur le plan socioéconomique de sa proximité au dynamique système métropolitain francilien (Mirloup, 2002 ; Allorant et al., 2020) mais ne constitue pas une métropole telle qu'on l'entend en géographie ou en urbanisme (Ascher, 1995; Lacour \& Puissant, 1999). La métropolisation évoquée dans cet article renvoie en effet à la généralisation d'un statut juridique français réservé en 2014 aux 10 principales villes de province ${ }^{\mathrm{ii}}$ avant d'être étendu en 2017 à 22 grandes agglomérations ${ }^{\text {iii }}$ (Dumont \& Chalard, 2007). Communauté d'agglomération depuis 2002, Orléans devient ainsi métropole le $1^{\mathrm{er}}$ mai $2017^{\mathrm{iv}}$.

Dans ce contexte institutionnel, que peut engendrer l'intervention métropolitaine en matière de santé ? La question se pose dans la mesure où la santé relève historiquement de l'État qui assure la formation des professionnels de santé, le financement des hôpitaux publics, la solvabilité de la demande de soin des patients, à travers l'assurance maladie, et la planification territoriale de l'offre, à travers le plan régional de santé. Néanmoins, l'administration locale ne s'est jamais désintéressée de ce champ d'action. Le rôle traditionnel du maire à propos de la salubrité publique a conduit à l'émergence d'un hygiénisme municipal qui s'est inscrit progressivement dans une logique de santé publique (Quesne, 1994 ; Barles, 1999), préfigurant l'approche globale de l'OMS sur cette dernière notion.

6 Au-delà de cet héritage historique, le contexte territorial a contraint l'échelon métropolitain à s'intéresser aux enjeux de santé. La situation du Centre-Val de Loire est préoccupante : il s'agit de la région française la plus sous-médicalisée. L'agglomération orléanaise en est elle-même affectée. Dépourvue de Centre hospitalier universitaire (CHU), Orléans est la seule des 13 capitales régionales et des 22 métropoles à être ainsi touchée par la désertification médicale (figure 1). La Communauté d'agglomération aujourd'hui Métropole - comptait 437 médecins généralistes, soit une densité de 159 professionnels pour 100000 habitants, densité en apparence moins alarmante que dans l'ensemble du Loiret (121) et du Centre-Val de Loire (130)v. Toutefois, la patientèle provient de territoires situés au-delà de la Métropole et de ses quelques 22 communes, ce qui aggrave les tensions ressenties par la population orléanaise. Cette situation 
préoccupante à plusieurs niveaux incite à une meilleure coopération en matière de santé non seulement entre la Métropole et les intercommunalités environnantes, mais encore entre la Métropole et les institutions compétentes (Agence régionale de santé ARS, collectivités territoriales, professionnels de santé).

Figure 1 : Orléans, seule métropole et capitale régionale de France dépourvue de Centre hospitalier universitaire

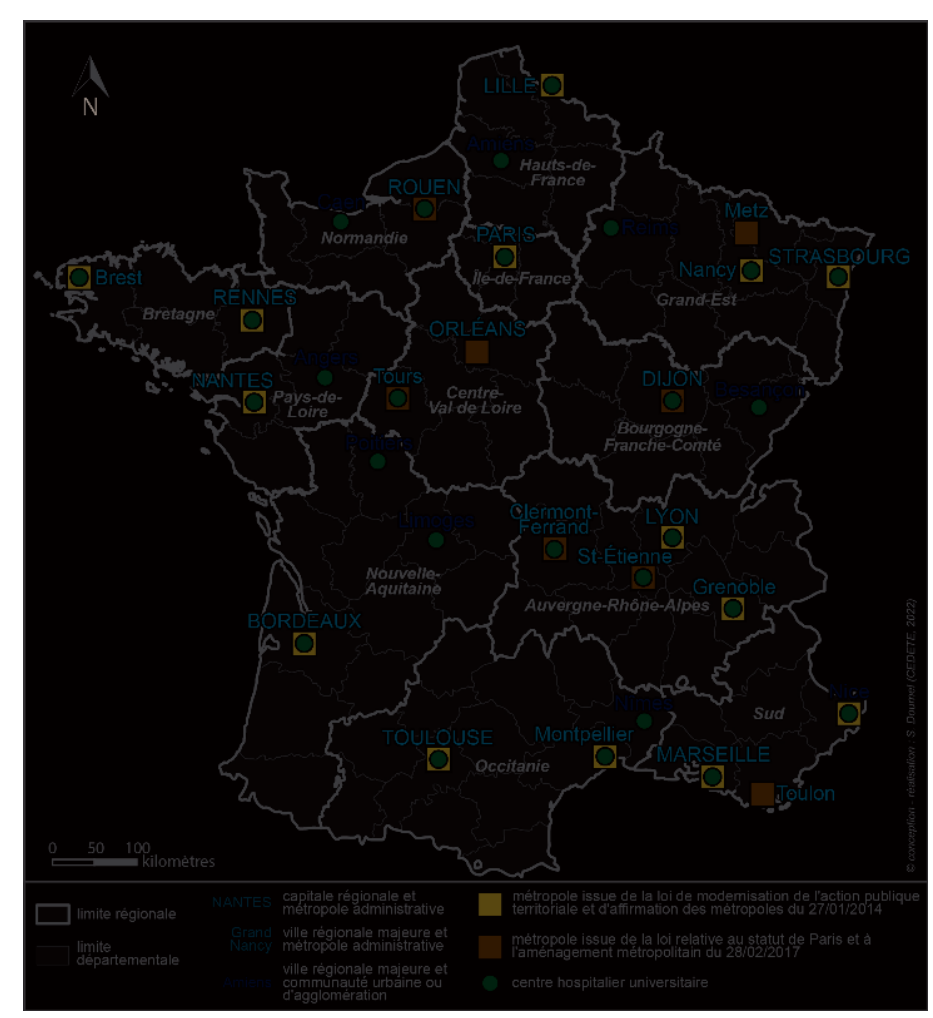

7 Les carences de l'offre de soins ont logiquement suscité des préoccupations et revendications de la part des électeurs. Aussi, le Conseil économique, social et environnemental régional Centre-Val de Loire (CESER) a été saisi par une pétition citoyenne sur la situation critique des hôpitaux dans le ressort régional. Les maires, en première ligne, ont tenté de répondre aux demandes de leurs administrés par la création de centres de santé ou de maisons de santé pluridisciplinaire (MSP). Cependant, l'ampleur des besoins et le caractère systémique de la crise dépassent très nettement l'échelon communal. C'est ainsi que le conseil de développement d'Orléans Métropole a créé un groupe de travail "Santé », centré sur les professionnels libéraux, et a présenté ses préconisations en février 2020, appelant à une meilleure articulation des interventions publiques.

De ce fait, l'insuffisance de l'offre locale de santé pose la question de l'attractivité de l'agglomération orléanaise dans l'installation de professionnels de santé, en effectifs suffisants pour assurer l'accès aux soins d'une population d'un bassin démographique et d'emploi dynamique. Son passage en métropole peut-il provoquer un changement de représentations à son bénéfice? Appartenir au club des métropoles est-il un levier efficace d'une politique de communication, de séduction et d'accueil de professionnels en majorité formés ailleurs? Les atouts de la métropole, parmi lesquels son niveau de vie médian élevé (le plus haut des principales "villes à une heure de Paris " "iv le second des 22 métropoles françaises), ses nombreux équipements et services, ses disponibilités 
foncières et immobilières, ses plus-values patrimoniales et paysagères, peuvent-ils être mieux valorisés grâce à ce statut nouveau ? L'accès à une offre supérieure de soins permettra-t-elle de replacer la métropole dans son réseau spatial fondamental privilégié, à l'interface entre la région parisienne et le Val de Loire ?

Face à ces nombreux questionnements, le passage en métropole constitue un moment décisif de l'adaptation du bloc communal aux enjeux de santé : mettre en œuvre de nouveaux dispositifs de l'action publique locale pour pallier les déficiences des politiques nationale et régionale, tout en recomposant les liens entre communes et intercommunalité. Si une nouvelle capacité d'action publique locale est manifestement en émergence, les entraves à son développement ne sont toutefois pas négligeables (Honta \& Basson, 2017).

\section{Les obstacles à l'intervention métropolitaine en matière d'offre de soins}

10 La capacité d'action de la Métropole en matière d'offre de soins peut se mesurer au regard de ses propres compétences sur le sujet et à l'aune de son intégration dans les réseaux d'acteurs pouvant influer sur l'ampleur de l'offre. Sur ces deux points, Orléans Métropole, sans être dépourvue de tout moyen d'action, fait face à des entraves certaines.

\section{Des compétences limitées et concurrencées}

11 Que peut impulser Orléans Métropole pour faire face à un déficit de l'offre de soins? Juridiquement, les compétences mobilisables sont assez limitées, d'autant qu'elles n'ont pas de caractère stratégique. À cet aspect se conjuguent de nombreuses initiatives concurrentes d'autres acteurs.

\section{L'absence de compétence stratégique}

12 La Métropole ne détient pas de compétence stratégique concernant l'offre de soins, c'est-à-dire qu'elle ne peut fixer, pour son périmètre, le cadre général de cette politique.

13 À ce titre, on peut partir du code général des collectivités territoriales (CGCT) qui énumère les compétences pouvant être exercées par les métropoles de droit commun, Orléans Métropole relevant de cette catégorie. D'une part, il y a les compétences exercées de plein droit par les métropoles, en lieu et place des communes membresvii. Aucune ne permet à la métropole d'organiser, sur son périmètre, l'offre de soins. D'autre part, on trouve les compétences qui peuvent revenir à la métropole par délégation, celle-ci prenant la forme d'un contrat pouvant être passé avec l'État, le département ou la région ${ }^{\text {viii }}$. Les compétences pouvant être déléguées par l'État ou le département à la métropole ne concernent jamais la santé. Ce constat doit être nuancé à propos de la région : d'après l'article L. 5217-2-V du CGCT, par convention passée avec la région, à la demande de celle-ci ou de la métropole, celle-ci exerce à l'intérieur de son périmètre, en lieu et place de la région, les compétences définies à l'article $\mathrm{L}$. 4221-1-1. Or, selon cet article, le conseil régional a compétence pour promouvoir le 
développement sanitaire de la région. Toutefois, la formule législative est floue et semble peu solide pour asseoir une compétence stratégique en matière de soins.

Assurément, c'est bien l'ARS qui va jouer un rôle majeur s'agissant de la planification stratégique de l'offre de soins. Celle-ci est contenue dans le plan régional de santé, qui définit les objectifs pluriannuels de l'ARS dans ses domaines de compétences ainsi que les mesures tendant à les atteindre ${ }^{\text {ix }}$. Ce plan donne lieu à des sectorisations, dont certaines peuvent concerner l'enjeu du déficit de l'offre de soins dans certaines zones. Par exemple, le schéma régional de santéx indique, dans le respect de la liberté d'installation, les besoins en implantations pour l'exercice des soins de premier recours et des soins de second recours mentionnés ${ }^{x i}$. Autre illustration, les zonages issus d'arrêtés pris par le directeur général de l'ARS. En particulier, celui-ci peut déterminer les zones caractérisées par une offre de soins insuffisante ou par des difficultés dans l'accès aux soins, pour les professions de santé et pour les spécialités ou groupes de spécialités médicales pour lesquels des dispositifs d'aide sont prévus. Dans ces zones sont mises en œuvre des mesures destinées à réduire les inégalités en matière de santé et à favoriser une meilleure répartition géographique des professionnels de santéxii.

Le constat est clair, sur l'aspect stratégique : fixer une répartition équilibrée de l'offre de soins et arrêter les mesures permettant de corriger les inégalités d'accès aux soins sont des prérogatives du ressort de l'ARS, non de la métropole.

\section{Des compétences d'exécution concurrentes}

Si le champ des compétences métropolitaines apparaît restreint, ce n'est pas uniquement $d u$ fait du droit applicable. Celui-ci ouvre d'autres possibilités aux métropoles, à propos de la mise en œuvre de la politique d'offre de soins. Cependant, les potentialités juridiques ne sont pas toujours exploitées, en raison des concurrences territoriales, d'autres acteurs pouvant agir par préférence à la Métropole.

On peut déjà le voir dans les relations entre Orléans Métropole et ses communes membres.

Les communes d'Orléans Métropole demeurent très actives à propos de l'enjeu de l'offre de soins. Clairement, la forte demande sociale pour l'accès aux soins, en particulier les médecins généralistes et spécialistes, oblige les communes à se saisir de ce sujet éminemment politique. La campagne pour les municipales de 2020 a été très révélatrice à cet égard, la santé apparaissant comme un enjeu majeur pour tous les candidats. Si l'on prend le seul exemple de la campagne relative à la ville-centre Orléans, les quatre candidats présents au second tour ont formulé des propositions en matière de santé parmi lesquelles :

- la création d'un centre de santé municipal (Serge Grouard, LR-UDI) ;

- l'attribution d'un médecin référent pour chaque Orléanais (Olivier Carré, DVD, LREMModem);

- l'installation d'une équipe de médecins salariés sur chaque quartier déficitaire (JeanPhilippe Grand, EELV) ;

- la désignation d'un référent santé dans chaque quartier pour faire le lien entre les professionnels de santé et les habitants (Baptiste Chapuis, PS-PC).

Avant même la campagne, les actions mayorales pour développer l'offre de soins sont nombreuses. On peut citer par exemple les ouvertures, annoncées début 2020, d'un centre de santé à Ingré, avec deux médecins salariés, et d'une nouvelle maison de santé 
à Saint-Jean-de-la-Ruelle (figure 2). Face à ces initiatives communales, Orléans Métropole n'est guère mise en avant, les maires, incarnant la figure du "maire entrepreneur ", revendiquant le lancement et la réussite des projets. Les seuls acteurs non municipaux évoqués sont l'ARS, qui doit donner son accord à l'ouverture des structures, voire la région, les communes se saisissant très vite de l'annonce du recrutement de médecins salariés par la région pour revendiquer la mise à disposition de praticiens pour leurs territoires. Naturellement, le risque patent est celui d'une concurrence entre communes, pour lancer des projets, attirer des praticiens, dans un contexte d'attrition des ressources publiques et du nombre de praticiens disponibles.

Figure 2 : politiques et équipements de santé rapportés à la carte de l'intercommunalité dans l'aire urbaine d'Orléans



Cet interventionnisme communal a, jusqu'à maintenant, conduit à un certain retrait du niveau intercommunal, alors que celui-ci peut potentiellement intervenir à deux titres.

Premièrement, la Métropole peut théoriquement contribuer à la régulation de la répartition de l'offre de soins sur son périmètre géographique, notamment pour arbitrer entre les communes le cas échéant. À cette fin, Orléans Métropole pourrait agir en recourant au CLS. Les CLS ont pour vocation de mettre en œuvre le plan régional de santéxiii. La loi indique que ces CLS peuvent être conclus par les collectivités territoriales et leurs groupements avec l'ARS. Orléans Métropole pourrait donc porter une telle convention. Ces CLS sont des instruments intéressants pour les collectivités souhaitant mener une politique publique d'offre de soins. En substance, leur objet porte sur la promotion de la santé, la prévention, les politiques de soins et l'accompagnement médico-social et social ${ }^{\mathrm{xiv}}$. Outre ce contenu, il faut souligner que les projets de santé des Communautés professionnelles territoriales de santé (CPTS) s'appuient sur les CLS, lorsqu'ils existent ${ }^{\mathrm{xv}}$. Cela signifie que les pouvoirs locaux, à travers la conclusion de CLS, peuvent influencer les professionnels de santé. Par rapport à cet outil, la situation 
d'Orléans Métropole est paradoxale : il n'existe pas de CLS métropolitain mais un CLS couvrant partiellement le périmètre de la Métropole (figure 2), le document étant porté par une commune membre, Fleury-les-Aubrais.

Deuxièmement, la Métropole dispose d'habilitations lui permettant de développer l'offre de soins, mais ces possibilités juridiques ne sont pas mises en œuvre, en particulier du fait de concurrences territoriales.

Ainsi, la loi prévoit que les métropoles peuvent créer et gérer des centres de santéxvi. Grâce à ces centres de santé, structures sanitaires de proximité, les collectivités territoriales peuvent contribuer de manière non négligeable à l'offre de soins dans leurs territoires. Au minimum, les centres de santé dispensent des soins de premier recours et, le cas échéant, de second recours et pratiquent à la fois des activités de prévention, de diagnostic et de soins, au sein du centre, sans hébergement, ou au domicile du patient. Ces centres peuvent assurer, le cas échéant, une prise en charge pluriprofessionnelle, associant des professionnels médicaux et des auxiliaires médicaux xvii. Outre ces activités de base, les centres de santé peuvent exercer des missions supplémentaires, si les collectivités les créant et gérant en décident ainsi ${ }^{\text {xiiii. }}$. Par exemple, les centres peuvent mener des actions de santé publique en vue de favoriser l'accès aux soins des personnes les plus vulnérables : ils peuvent contribuer à la permanence des soins ambulatoires, pratiquer des IVG...

Cependant, cet instrument n'est pas mobilisé par Orléans Métropole. D'un côté, pour ne pas porter ombrage aux projets menés par les communes membres. De l'autre, en raison du fort volontarisme de la région Centre-Val de Loire. La collectivité a en effet lancé un Plan urgence santéxix, visant à corriger les faiblesses en ce domaine par la poursuite de cinq objectifs :

- la formation des professionnels de santé ;

- le renforcement du maillage de l'ensemble du territoire ;

- la conduite de politiques de régulation des inégalités sociales et territoriales pour un accès aux soins pour tous ;

- le financement de projets de MSP pour lutter contre la désertification médicale et contre l'isolement des professionnels de santé ;

- le financement de l'aménagement numérique du territoire qui doit permettre d'optimiser les pratiques de télémédecine et de télé-enseignement.

L'effort régional est important. Par exemple, la Région indique avoir investie $20 \mathrm{M} €$ pour la création de MSP sur l'ensemble de son territoire. Autre illustration, de manière très innovante, la Région a annoncé, en novembre 2019, le recours à un nouvel instrument, le salariat de médecins, le président François Bonneau annonçant le recrutement de 150 médecins.

\section{Une absence d'inscription systématique dans les réseaux de coopération}

26 Au-delà des compétences qui lui permettent d'agir en son nom propre, la capacité d'action de l'institution métropolitaine dépend aussi de son intégration dans les réseaux locaux et régionaux de coopération qui influent sur l'offre de soins. En d'autres termes, se pose la question de l'implication de la métropole dans les organisations, structures ou instances regroupant des acteurs afin de les faire dialoguer et/ou agir, notamment sur l'enjeu de l'offre de soins. L'intégration dans ces réseaux peut générer 
de nouvelles ressources en faveur de la métropole ou permettre à celle-ci d'influencer la prise de décision dans un sens lui étant favorable. Dans cette optique, la réalité de la position d'Orléans Métropole apparait largement perfectible.

\section{Une association non obligatoire au niveau régional}

Sur le plan juridique, la métropole n'est pas obligatoirement associée à toutes les instances régionales regroupant divers acteurs afin de les faire intervenir sur la question de l'offre de soins. L'absence de contrainte juridique n'empêche pas toujours l'intégration d'une métropole à de telles instances: l'implication métropolitaine dépend alors du volontarisme des acteurs locaux. Toutefois, celui-ci n'est pas systématiquement caractérisé. Tout cela conduit concrètement Orléans Métropole à ne pas être associée à certaines de ces instances, ce que l'on peut illustrer.

Le premier exemple concerne les établissements de santé. Pour tout territoire, et a fortiori dans un territoire souffrant d'une insuffisance de l'offre de soins, les cliniques et hôpitaux publics sont fondamentaux. Pour autant, le droit ne reconnaît pas de rôle conséquent aux métropoles administratives s'agissant de leur création et déploiement. Ainsi, toute création relève de la compétence de l'ARS ${ }^{\mathrm{xx}}$. Avant de statuer, cette dernière doit certes consulter la commission spécialisée de la conférence régionale de la santé et de l'autonomie compétente pour le secteur sanitaire ${ }^{\mathrm{xxi}}$. D'après la loi, sont notamment représentés au sein des collèges de la conférence les collectivités territoriales, les usagers et associations œuvrant dans les domaines de compétence de l'ARS, les conseils territoriaux de santé, les organisations représentatives des salariés et des professions indépendantes, les organisations professionnelles d'employeurs représentatives au niveau national et interprofessionnel, les professionnels du système de santé, les organismes gestionnaires des établissements et services de santé et médico-sociaux, les organismes de protection sociale ${ }^{\mathrm{xxii}}$. Ainsi, la loi ne prévoit pas une présence obligatoire des métropoles parmi les membres de cette conférence. L'absence de contrainte juridique a donc pour effet, pour le cas de la conférence du Centre-Val de Loire, que ni Orléans Métropole, ni Tours Métropole Val de Loire n'en sont membresxxiii.

Le second exemple est relatif à une innovation portée par la région Centre-Val de Loire. Celle-ci, pour amplifier encore ses efforts en faveur de l'offre de soins, a constitué un groupement d'intérêt public (GIP) dénommé « GIP Pro Santé Centre-Val de Loire ». La convention constitutive de cette personne publique a été conclue le 11 juin 2020 par ses membres fondateurs : la région Centre-Val de Loire, l'Association de soin et de services à domicile - hospitalisation à domicile (ASSAD-HAD) et l'Association pour l'emploi des cadres (APEC). D'après sa convention constitutive, le GIP a pour objet principal de porter la création et l'animation de centres de santé, lesquels permettront de recruter des professionnels de santé, médecins généralistes en particulier conformément aux dispositions des articles L. 6323-1 et suivants du code de la santé publique. Pour atteindre cette finalité générale, deux missions principales lui sont attribuées :

- porter la création et l'animation de centres de santé, lesquels permettent de lui donner compétence pour recruter les professionnels de santé, principalement les médecins généralistes au cœur du projet ;

- identifier en lien avec l'ARS les secteurs géographiques prioritaires pour leur implantation, territoires dans lesquels l'accès de la population aux soins est inexistant, insuffisant ou inadapté. 
Un tel champ d'action est très sensible pour les collectivités locales et leurs groupements, les choix régionaux pouvant avoir de forts impacts sur la situation de l'offre de soins pour un territoire donné. En anticipation, la convention constitutive précise que sur chaque secteur géographique identifié, la création du centre de santé et de ses éventuelles antennes, sera étudiée en lien étroit avec l'ARS et les acteurs locaux : collectivités territoriales, établissements et acteurs de la santé, professionnels de santé libéraux, représentés via l'Union régionale des professionnels de santé (URPS) et les CPTS pour une complémentarité optimale avec l'offre libérale. Au-delà de cet énoncé de principe, le GIP est structuré en différents collèges, répartissant les membres du groupement à l'Assemblée Générale. Ainsi, on distingue un collège ${ }^{\circ} 2$ réunissant les collectivités territoriales (autres que la Région) et leurs groupements. Cependant, Orléans Métropole n'en est pas membre.

\section{Une association non obligatoire au niveau local}

31 Par ailleurs, Orléans Métropole n'est pas systématiquement intégrée aux réseaux locaux pouvant avoir un impact sur l'offre de soins. Deux illustrations peuvent l'étayer, l'un sur l'angle hospitalier (Keller, 2020), l'autre s'agissant des rapports interterritoriaux (Vannier, 2010).

32 En premier lieu, la Métropole apparaît peu présente s'agissant des groupements hospitaliers, dits groupes hospitaliers de territoire (GHT). Ceux-ci ont deux fonctions principales :

- définir et mettre en œuvre une stratégie de prise en charge commune et graduée du patient, dans le but d'assurer une égalité d'accès à des soins sécurisés et de qualité ;

- rationaliser les modes de gestion par une mise en commun de fonctions ou par des transferts d'activités entre établissements ${ }^{x i v}$.

Le GHT, au vu de ces fonctions, est clairement un instrument de rationalisation et d'économie. Son impact potentiel sur l'offre de soins d'un territoire apparait potentiellement considérable, d'où l'intérêt pour une Métropole de s'intéresser à ces groupements. Pour autant, c'est l'ARS qui reste le seul décideur concernant la formation des GHT, en approuvant leurs conventions constitutives ${ }^{\mathrm{xx}}$. On a vu précédemment l'absence d'association systématique des métropoles aux organismes consultatifs relevant de l'ARS, notamment la conférence régionale de la santé et de l'autonomie : l'influence interne de la métropole ne peut être que très relative. Au-delà de la constitution du GHT, son fonctionnement ordinaire ne permet guère à la métropole de mieux faire entendre sa voix et ses analyses. Certes, la convention constitutive du GHT doit impérativement prévoir un comité territorial des élus locaux. Ce comité est chargé d'évaluer les actions mises en œuvre par le groupement pour garantir l'égalité d'accès à des soins sécurisés et de qualité sur l'ensemble du territoire du groupement. À ce titre, il peut émettre des propositions et est informé des suites qui leur sont données. Si le rôle du comité n'est pas négligeable, on note qu'aucune disposition n'impose une représentation d'élus métropolitains et que rien ne donne une prééminence à ces derniers, et donc, à la métropole.

En second lieu, les rapports interterritoriaux d'Orléans Métropole sont à ce jour extrêmement limités. On peut notamment relever l'absence de coordination interterritoriale des deux CLS de l'aire urbaine orléanaise. À ce titre, il faut indiquer que l'ensemble des communautés de communes entourant Orléans Métropole sont 
adhérentes d'un seul et unique CLS, le CLS des "Territoires Ruraux de l'Orléanais » (figure 2), regroupant le Pôle d'équilibre territorial et rural «Forêt d'Orléans-LoireSologne » (3 Communautés de communes, dont 49 communes), le Pôle d'équilibre territorial et rural et Pays «Loire Beauce » (2 Communautés de communes dont 48 communes) ainsi que la Communauté de communes des "Portes de Sologne »xvi (7 communes). En outre, on a vu précédemment que le CLS de l'agglomération orléanaise ne regroupe pas toutes les communes membres de la Métropole, celle-ci ne pilotant pas le dispositif. Ces particularismes privent Orléans Métropole de la possibilité de jouer un rôle à l'égard des territoires voisins, par exemple via un mécanisme d'inter-CLS. Cette position médiatrice serait pourtant utile. D'une part, car les difficultés sont communes, les territoires ayant en partage un déficit de l'offre de soins, les manques étant même aggravés au-delà des limites métropolitaines. D'autre part, parce que la Métropole et ses voisins entretiennent des relations fonctionnelles, par exemple du fait de la présence du CHRO et des spécialistes sur le périmètre métropolitain.

En apparence, la Métropole semble mal armée pour intervenir sur ce champ, tant en termes de ressources juridiques que d'insertion dans les cadres de coopération pertinents. Concurrencée par d'autres acteurs, pas systématiquement associée aux instances décisionnelles, dotée d'habilitations mises en oeuvre de manière relative : ces éléments rappellent le particularisme du champ nouvellement investi par la métropole. Effectivement, il a déjà été souligné que le champ de la santé se caractérisait par une prolifération d'acteurs, de dispositifs, de périmètres, générant une puissante complexité et un besoin de clarification dans la répartition des rôles (Le Goff, 2012 ; Bourgueil, 2017). Dans cette toile à l'intelligibilité limitée, la métropole ne peut s'intégrer qu'avec difficultés. Pour autant, la créativité métropolitaine permet aussi de dépasser certaines lacunes instrumentales pour bâtir une véritable capacité d'action au bénéfice de la Métropole.

\section{Les leviers de l'intervention métropolitaine en matière d'offre de soins}

Pour contribuer au développement de l'offre de soins sur son périmètre, la métropole orléanaise utilise deux types de ressources. Premièrement, elle mobilise des compétences connexes qu'elle va exploiter en faveur de l'offre de soins. Deuxièmement, Orléans Métropole se dote d'une compétence nouvelle et originale en matière de régulation de l'offre.

\section{La mobilisation de compétences connexes en faveur de l'offre de soins}

37 Sans être dotée explicitement d'une habilitation légale de développement de l'offre de soins, Orléans Métropole cherche à améliorer celle-ci, en usant de compétences connexes, soit pour attirer des praticiens de santé, soit, de manière plus innovante, pour mener des actions de soin. 


\section{Les compétences connexes mobilisées pour attirer des praticiens de santé}

portant à leur connaissance les opportunités professionnelles et personnelles à venir s'installer dans le territoire, présenté comme une source de qualité de travail et de vie. De manière très concrète, cela amène le chargé de mission à des actions de prospection dans les salons, congrès et réseaux médicaux. De même, la mise en avant du territoire passe par des opérations visant à le rendre visible sur l'espace public, qu'il s'agisse de l'espace physique ou numérique. Ainsi, en 2019, une importante campagne d'affichage a été lancée, dans le métro parisien mais aussi via le mobilier urbain, en région parisienne ainsi qu'à proximité des CHU et UFR de médecine situés à deux ou trois heures d'Orléans : à Amiens, Angers, Caen, Clermont-Ferrand, Dijon, Limoges, Poitiers et Reims (figure 3). Dans la sphère numérique, des comptes sur les réseaux sociaux relaient cette stratégie d'attractivité médicale d'Orléans Métropole. Les démarches ne sont pas uniquement conduites par ce seul chargé de mission. Celui-ci a également pour rôle d'animer le "Cercle des Médecins Ambassadeurs d'Orléans Métropole ». Autrement dit, des médecins déjà présents dans le périmètre de la Métropole sont sollicités pour participer aux actions valorisant le territoire auprès de leurs confrères, soit en validant des outils de communication envisagés par la Métropole, soit en s'adressant directement à eux, avec l'idée que des médecins peuvent convaincre plus aisément des médecins de s'installer que des personnes extérieures à cette corporation très soudée. Cette philosophie s'est étendue à d'autres praticiens, en particulier les internes, pour amener ceux-ci à envisager leur lieu d'exercice futur dans la métropole orléanaise.

D'autre part, sont proposés un accueil et un accompagnement à l'installation personnelle, familiale et professionnelle dans le territoire métropolitain. À ce titre, le chargé de mission suit tous les sujets afférents à l'installation des praticiens, pour les aider à trouver des logements, inscrire leurs enfants dans les écoles, conseiller les conjoints dans leurs recherches de postes... Au-delà de la dimension personnelle et familiale, l'accompagnement touche aussi à la sphère professionnelle, par exemple via le financement de l'abonnement auprès d'une plateforme de prise de rendez-vous en ligne. Plus globalement, la Métropole contribue à développer un bouquet de services adapté aux besoins et attentes de chaque praticien, particulièrement les médecins généralistes, en les déchargeant des démarches administratives, et en leur facilitant la recherche de secrétariat et d'assistance informatique. 
Figure 3 : Le pari d'Orléans Métropole : pallier les carences de l'offre de soin en attirant les professionnels de santé grâce à la qualité de vie et de travail

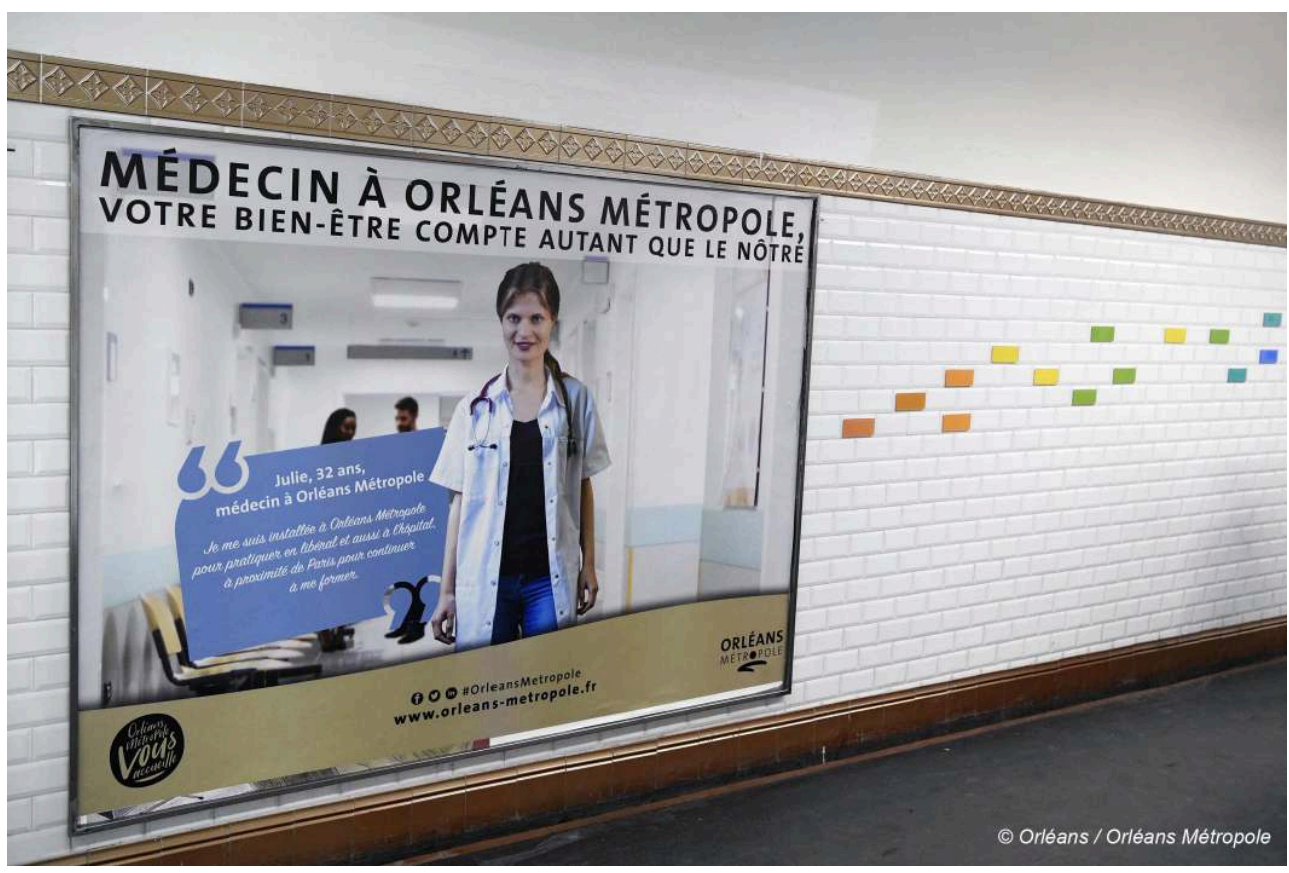

Dans un second temps, on peut souligner que les capacités d'action d'Orléans Métropole sur le plan foncier et immobilier peuvent aussi être mises au service de la politique générale d'attractivité médicale du territoire. À ce titre, on peut citer l'exemple de la société d'économie mixte patrimoniale (SEMPAT) Orléans Val de Loire. La société, contrôlée à $36 \%$ par la Métropole et à $34 \%$ par la ville-centre Orléans, est présentée sur le site internet de la Société d'économie mixte pour le développement orléanais comme " une foncière immobilière qui investit dans des actifs immobiliers favorisant le développement économique d'Orléans Métropole et du Loiret »xvii. Plus précisément, il est indiqué que «les investissements réalisés par la SEMPAT Orléans Val de Loire portent sur des projets de construction, de réhabilitation, de rénovation, d'acquisition de biens immobiliers utiles au développement économique du territoire. Ces biens sont destinés à un usage locatif dans le domaine industriel, commercial, touristique, artisanal ou de services. La SEMPAT Orléans Val de Loire se consacre à la gestion, l'exploitation et l'entretien des immeubles bâtis ». Dans ce cadre, la SEMPAT est intervenue pour le lancement de la quatrième MSPxxviii présente sur le territoire de la commune d'Orléans, dans le centre-ville, en janvier 2021. Cette nouvelle MSP se trouve sur le périmètre de la zone d'aménagement concerté Carmes-Madeleine, au sein du prestigieux Hôtel-Dieu de l'hôpital Madeleine, aujourd'hui désaffecté. La SEMPAT a œuvré à la réhabilitation et à la rénovation de l'espace dédié à la MSP, pour permettre son ouverture.

\section{Les compétences connexes mobilisées pour mener des actions de soins}

43 Juridiquement, les autorités territoriales sont chargées de veiller à la sécurité et à la protection de la santé des agents placés sous leur autoritéxxvix. Cette obligation générale se traduit par la nécessité, pour les autorités territoriales, de créer des services de médecine préventive. Une originalité d'Orléans Métropole est que ce service est métropolitain. En partant de cette compétence obligatoire, relative à la santé des 
agents, il a été possible d'innover pour mener une action de soins et de recherche en pleine crise sanitaire, via le dispositif « Covidor ».

En substance, « Covidor » est une étude épidémiologique, conduite sous la direction du Docteur Serreau, praticien hospitalier et habilité à diriger des recherches, directeur du service de médecine préventive d'Orléans Métropole. Le projet a réuni celle-ci, mais également la communauté de communes voisine «Terres du Val de Loire » et la région Centre-Val de Loire. Sur le fond, l'étude porte sur la séroprévalence des agents publics des trois collectivités, pour mesurer leur exposition à la COVID-19. Plus de 2000 agents ont ainsi déjà été testés et l'étude épidémiologique a débouché sur plusieurs publications scientifiques. Ce dispositif constitue une action opérationnelle concrète en matière de soins, sous l'angle du dépistage.

\section{L'affirmation progressive d'une compétence métropolitaine en matière d'offre de soins}

Orléans Métropole développe ses capacités d'action en matière d'offre de soins, au-delà de l'utilisation de ses compétences connexes. C'est une compétence de coordination de cette offre qui est désormais consacrée, l'institution métropolitaine se dotant d'un pouvoir d'expertise lui permettant de l'exercer.

\section{La construction d'un rôle de régulateur porté politiquement}

46 À défaut de rôle stratégique concernant l'offre de soins, Orléans Métropole agit au moment de la mise en œuvre de la politique, dans une fonction de régulation. En effet, la Métropole s'est dotée, par ses statuts, d'une attribution lui permettant de s'affirmer : depuis novembre 2018, elle est compétente pour la « coordination des projets d'installation et de maintien des professionnels de santé ». Manifestement, Orléans Métropole a su profiter des souplesses offertes par le droit de l'intercommunalité à propos de la répartition des compétences entre les niveaux communaux et intercommunaux. Plus précisément, elle a bénéficié de l'existence des compétences dites facultatives ou supplémentaires, c'est-à-dire celles qui sont transmises librement par les communes, en dehors des contraintes légales. C'est dans cette catégorie que s'inscrit la compétence de coordination d'Orléans Métropole.

$\mathrm{Au}$ premier abord, cette compétence semble assez limitée: une fonction de coordination n'est pas une fonction de répartition quantitative des praticiens entre les communes. En d'autres termes, la métropole n'impose pas la localisation des professionnels de santé, qui bénéficient de toute façon d'une liberté d'installation ${ }^{\mathrm{xxx}}$ : elle intervient plutôt comme médiatrice entre les communes pour rechercher la distribution spatiale la plus efficiente possible des professionnels de santé. Ainsi, les communes conservent la possibilité d'initier une politique visant à attirer ou maintenir sur leurs territoires des praticiens, en dépit des doutes, depuis longtemps exprimés, sur l'efficacité de ces mesures incitatives. La métropole vise à articuler au mieux ces politiques communales, de manière à favoriser l'accès aux soins sur l'ensemble du périmètre métropolitain, également pour éviter les concurrences entre communes. Une forme de magistrature d'influence rappelant l'office des autorités de régulation.

Naturellement, l'absence de pouvoir contraignant rend l'office métropolitain dépendant des négociations avec les communes et de la formation de consensus. Cela 
rend sa portée incertaine, dans un contexte où la demande médicale des populations est pressante, ce qui peut conduire à un certain individualisme communal.

Toutefois, l'intervention nouvelle d'Orléans Métropole à propos de l'offre de soins ne doit pas être sous-estimée. D'abord, car c'est une première très innovante, en-dehors des cadres juridiques traditionnels qui n'ont jamais fait de l'intercommunalité un acteur majeur des politiques de soins. Celle-ci est traditionnellement appréhendée comme un instrument en faveur de l'aménagement et du développement économique. Ensuite, il faut bien voir les potentialités de cette première implication métropolitaine qui donnera peut-être lieu à une compétence plus développée dans le futur en matière d'offre de soins. Il est d'ores et déjà envisagé que le pilotage du CLS se fasse désormais à l'échelle métropolitaine. Si l'on prend d'autres compétences, comme l'urbanisme par exemple, le mouvement d'affirmation intercommunale est toujours progressif. Enfin, la nouvelle compétence métropolitaine s'est traduite par un portage politique inédit à la suite des élections survenues à l'été 2020. Il y a désormais un vice-président dédié à la «coordination de la politique de santé métropolitaine ». Cette innovation marque la légitimation métropolitaine en matière de santé.

\section{Le développement d'un pouvoir d'expertise}

Pour s'affirmer quant à la politique d'offre de soins, au-delà des compétences et du portage politique, il faut pouvoir démontrer son expertise pour asseoir la légitimité de l'intervention métropolitaine. À ce titre, deux initiatives permettent le développement du pouvoir d'expertise d'Orléans Métropole.

51 En 2019, Orléans Métropole a rejoint le programme « POPSU Métropole », c'est-à-dire la "plateforme d'observation des projets et stratégies urbaines» dédiée aux métropoles. La philosophie POPSU est de réunir des élus, des administrateurs et des chercheurs pour réfléchir à la question métropolitaine. Plus précisément, Orléans Métropole a rejoint le programme au moment où celui-ci a lancé un thème de recherche sur « La Métropole et son voisinage ». Les échanges entre élus, administrateurs et chercheurs ont conduit à proposer le sujet de la santé dans une perspective interterritoriale, les relations entre Orléans Métropole et ses territoires voisins. Dans un espace orléanais confronté à un déficit de l'offre de soins, la santé est apparue comme une priorité partagée, permettant à des territoires aux rapports distendus de nouer des collaborations inédites. Les résultats de la recherche doivent nourrir l'expertise d'Orléans Métropole et pourront influencer les prochaines étapes de sa politique de santé.

52 Au préalable, Orléans Métropole a initié fin 2018 un Observatoire de la santé, sous l'égide de TOPOS ${ }^{\mathrm{xxx}}$, l'Agence d'urbanisme des territoires de l'Orléanais. À son origine, un conflit entre Orléans Métropole et l'ARS : en cherchant à contester des choix arrêtés par l'ARS, la métropole a réalisé ses lacunes en termes d'expertise et de données disponibles. En effet, l'ARS, pour l'allocation des moyens et des équipements permettant de répondre à la demande de soins, procède à des classifications des territoires, l'urgence des situations fondant la distribution des ressources. Précisément, Orléans Métropole contestait une taxinomie retenue par l'ARS qui avait pour effet de ne pas lui permettre d'accéder aux ressources estimées nécessaires pour faire face aux besoins du territoire. Cette dissonance a fait prendre conscience à la Métropole qu'elle ne disposait pas du pouvoir d'expertise nécessaire pour pouvoir discuter les arbitrages retenus par l'ARS, qui concentre les données permettant de répartir les ressources 
entre les territoires. Orléans Métropole a donc souhaité bâtir une capacité autonome de collecte et d'analyse de données concernant la santé, entendue de prime abord comme l'offre de soins, avant d'être élargie à tout ce qui contribue au bien-être. L'Observatoire collectera des informations, produira des études pour la Métropole et ses voisins.

\section{En attendant la synergie des collectivités : la métropole avec les autres}

53 La Métropole ne pourra tout faire seule : sa compétence santé est embryonnaire et ne dissimule pas la réalité de la prééminence de l'État et de ses décisions pour comprendre la réalité de l'offre de soins dans les territoires. Ainsi, la loi Santéxxxii n'a "supprimé » le numerus clausus, qui resserre avec malthusianisme depuis près d'un demi-siècle les effectifs de professionnels de santé formés, qu'en apparence. En réalité, le pouvoir de décision a été transféré à chaque doyen de faculté de médecine, ce nouveau numerus apertus ne réservant à l'ARS et aux collectivités territoriales concernées qu'un avis consultatif. Or, l'ardente obligation de former davantage de médecins et de mieux les répartir sur le territoire demeure de la responsabilité de l'État. À l'occasion des prochaines échéances électorales, il serait souhaitable que les questions soient posées dans le débat public d'une contrainte temporaire de première installation des jeunes praticiens, formés puis rémunérés aux frais de la nation, au cœur des déserts médicaux, avec pour contrepartie une amélioration de la qualité de vie au travail des internes, clés de voûte, avec les médecins étrangers, d'un système hospitalier français à bout de souffle.

Ne serait-il pas plus efficace et démocratique de confier davantage de responsabilités aux élus des territoires, à l'échelle régionale ou départementale, au détriment du " pouvoir médical » mandarinal et ministériel, afin de mieux articuler la politique de l'ARS et les interventions des collectivités? Dans cette perspective, les institutions métropolitaines pourraient-elles se voir reconnaître un pouvoir de régulation à l'échelle locale?

55 La crise COVID a souligné l'intérêt de coordonner les acteurs hospitaliers publics et privés et les professionnels de ville. Néanmoins, en dépit des commissions de l'ARS et des parfois timides ou incomplets CLS, les instances non seulement de dialogue mais de construction de décisions communes opérationnelles sur un territoire, un bassin de vie, font défaut. Alors que bien des institutions métropolitaines ou autres structures intercommunales restent dominées par le jeu d'une gouvernance verrouillée par les maires, la difficulté à sortir des rivalités entre communes milite pour de véritables conférences des territoires proactives. Pour sortir de l'entre soi des élus ou des professionnels, le rôle de pression de la société civile organisée (conseils de développement, CESER) forme un levier à mobiliser pour témoigner de "l'intérêt à agir » sur la pression des urgences ressenties par l'opinion publique. Certaines erreurs manifestes d'appréciation de l'intérêt public local auraient ainsi pu être évitées, telles les coupures artificielles de solidarités territoriales au nom d'une conception rigide des " frontières régionales "xxxiii, ou d'un découpage départemental des GHT, peu conforme aux complémentarités de terrain.

L'organisation opérationnelle et proactive de ces différents acteurs en réseau, à partir des territoires de démocratie sanitaire, offrira sans doute l'opportunité de mieux écouter et prendre en compte tant les besoins des professionnels de santé que les 
aspirations des usagers d'un territoire. Ainsi, les conseils territoriaux de santé pourraient constituer l'outil de partage des éléments de connaissance et d'approche fine des enjeux territoriaux de santé et de bien-être à travers un diagnostic territorial partagé (Thorigny \& Duchesne, 2018, 2020). Identifier et hiérarchiser les enjeux de santé après avoir mené des enquêtes sociologiques et animé des ateliers participatifs mais aussi mettre en cohérence l'ensemble des projets de l'ARS, voilà l'enjeu majeur. Cette coopération territoriale de proximité apparaît seule à même de dépasser les limites institutionnelles et d'améliorer la protection de la santé de la population.

Par exemple, pour la protection de la santé des étudiants, dont la pandémie a révélé la grande fragilité, la prévention des conduites addictives et à risque, la santé mentale, l'alimentation et les pratiques sportives mobilisent des acteurs aussi nombreux que mal coordonnés: Centres régionaux des œuvres universitaires et scolaires, Services universitaires de médecine préventive et de promotion de la santé, Métropole, ville, région, université, ARS, mutuelles... L'institution métropolitaine, par la transversalité de ses compétences qui la place dans tous les réseaux d'acteurs, pourrait être un acteur clé en la matière. L'articulation des coopérations locales, nouvelle frontière du développement de la capacité d'action métropolitaine en matière de santé ?

\section{BIBLIOGRAPHIE}

Allorant, P., Dournel, S., Eddazi, F., \& Guérit, F. (2021). La métropole par la santé ? Coopérations dans les territoires de l'Orléanais. Autrement.

Allorant, P., Guérit, F., \& Dournel S. (2020). Orléans, faire la "métropole intense" ? In C. Demazière, X. Desjardins \& $\mathrm{O}$. Sykes (dir.), La gouvernance des métropoles et des régions urbaines. Des réformes institutionnelles aux coopérations territoriales (pp. 249-267). Plan urbanisme construction architecture.

Ascher, J.-F. (1995). Métapolis ou l'avenir des villes, éd. Odile Jacob.

Barles, S. (1999). La ville délétère. Médecins et ingénieurs dans l'espace urbain : XVIIIe-XIXe siècle. Champs vallon.

Bourgueil, Y. (2017). L'action locale en santé : rapprocher soins et santé et clarifier les rôles institutionnels ?, Sciences sociales et santé, 35, 1, 97.

Bourgueil, Y., Mousquès, J., Ayden Tajahmadi A. (2006). Comment améliorer la répartition géographique des professionnels de santé ? Les enseignements de la littérature internationale et des mesures adoptées en France, Institut de recherche et documentation en économie de la santé. Rapport 534.

Cardoux, J.-N. \& Daudigny, Y. (2017). Rapport d'information sur les mesures incitatives au développement de l'offre de soins primaires dans les zones sous-dotées, Paris Sénat.

Dab., W., \& Roussel, I. (2001). L'air et la ville : les nouveaux visages de la pollution atmospéhrique. Hachette Littératures. 
Dumont, G.-F., \& Chalard, L. (2007). Les Métropoles régionales intermédiaires en France : quelle attractivité? Datar / La Documentation française.

Honta, M., \& Basson, J.-C. (2017). La fabrique du gouvernement métropolitain de la santé, Gouvernement et action publique, 6, 63-82.

Jomier, B., \& Surel, L. (2017). La santé dans les grandes métropoles, Les Tribunes de la Santé, 56, 29-34.

Keller, C. (2020). L'établissement de santé à l'épreuve de la coopération interhospitalière. Thèse de doctorat en droit. Université Rennes 1.

Lacour, C., \& Puissant, S. (1999), La métropolisation. Croissance, Diversité, Fractures, Anthropos.

Larrouy-Castera, X., \& Ourliac, J.-P. (2004). Risques et urbanisme : risques naturels, risques technologiques, prévention, responsabilités. Le Moniteur.

Le Goff, E. (2012). Les Villes-Santé en Bretagne : quels choix de gestion et d'aménagement des espaces? Thèse de doctorat en Géographie. Université Rennes 2.

Mirloup, J. (dir.) (2002). Régions périmétropolitaines et métropolisation. Presses Universitaires d'Orléans.

Pigeon, P. (2012). Paradoxes de l'urbanisation : pourquoi les catastrophes n'empêchent-elles pas l'urbanisation? L'Harmattan.

Pigeon, P. (2007). L'environnement au défi de l'urbanisation. Presses universitaires de Rennes.

Quesne, L. (1994). De l'insalubrité à l'hygiénisme. Émergence politique d'une problématique environnementale : l'exemple du Mans. Thèse de doctorat en géographie. Université du Maine.

Salomon-Cavin, J. (2005). La ville mal aimée. Représentations anti-urbaines et aménagement du territoire en Suisse : analyse, comparaisons, évolution. Presses polytechniques et universitaires romandes.

Thorigny, M., \& Duchesne, V. (2020). Quelle place pour l'expression citoyenne dans la définition de la politique régionale de santé ? Une analyse en région Centre-Val de Loire. In Population, temps, territoires : actes du 5 e colloque du Collège international des sciences territoriales (pp. 340-345).

Thorigny, M., \& Duchesne, V. (2018). Les représentations des territoires dans le cadre de la refonte du Projet Régional de Santé - L'exemple de la région Centre-Val de Loire. In Représenter les territoires : actes du 4 e colloque du Collège international des sciences territoriales (pp. 340-345).

Vannier, M. (2010). Le pouvoir des territoires : essai sur l'interterritorialité, Economica.

Veltz, P. (2014). Mondialisation, villes et territoires. Presses universitaires de France.

\section{NOTES}

i. À ce sujet, consulter l'entrevue de Laurent Davezies sur le site de la Gazette des communes (https://www.lagazettedescommunes.com/692017/le-covid-19-adavantage-frappe-les-metropoles-que-la-ruralite/) ainsi que le dossier thématique de la revue Géoconfluences «Mondialisation et démondialisation au prisme de la pandémie de Covid-19. Le grand retour de l'espace, des territoires et du fait politique » (http:// geoconfluences.ens-lyon.fr/actualites/eclairage/covid19-mondialisationdemondialisation). 
ii. La loi du 16 décembre 2010 de réforme des collectivités territoriales instaure la métropole en tant que nouvelle catégorie d'établissement public de coopération intercommunale à fiscalité propre. La loi du 27 janvier 2014 de modernisation de l'action publique territoriale et d'affirmation des métropoles (MAPTAM) en renforce le rôle et en précise les statuts.

iii. L'élargissement de l'EPCI « métropole » à 22 agglomérations françaises est associé à la loi relative au statut de Paris et à l'aménagement métropolitain du 28 février 2017.

iv. Notons qu'Orléans était préalablement communauté urbaine pour une durée de 4 mois. Tours, autre métropole de la région Centre - Val de Loire, avait suivi le même processus.

v. Chiffres du diagnostic local de santé conduit par l'observatoire régional de santé fin 2015, en vue de la conclusion du Contrat local de santé (CLS).

vi. Les principales villes à une heure de Paris renvoient à Amiens, Le Mans, Orléans, Reims, Rouen, Tours et Troyes.

vii. Article L. 5217-2-I du code général des collectivités territoriales.

viii. Article L. 5217-2 II à V du code général des collectivités territoriales

ix. Article L. 1434-1 du code de la santé publique.

x. Article L. $1434-2-2^{\circ}$ du code de la santé publique.

xi. Article L. $1434-3-\mathrm{I}-1^{\circ}$ du code de la santé publique.

xii. Article L. 1434-4 du code de la santé publique.

xiii. Article L. 1434-10-IV du code de la santé publique.

xiv. Article L. 1434-10-IV du code de la santé publique.

xv. Article L. 1434-10-IV du code de la santé publique.

xvi. Article L. 6323-1-3 du code de la santé publique.

xvii. Article L. 6323-1 du code de la santé publique.

xviii. Article L. 6323-1-1 du code de la santé publique.

xix. URL : https://www.centre-valdeloire.fr/agir/favoriser-le-bien-etre-la-sante/lasante-au-coeur-des-priorites-de-la-region

xx. Article L. 6122-1 du code de la santé publique.

xxi. Article L. 6122-9 du code de la santé publique.

xxii. Article L. 1432-4 du code de la santé publique.

xxiii. Arrêté nº 2020-DSTRAT-0014.

xxiv. Article L. 6132-1 du code de la santé publique.

xxv. Article L. 6132-2 du code de la santé publique.

xxvi. La Communauté de communes Portes de Sologne se substitue au Pays Sologne Val Sud qui a été dissous le 31 décembre 2018.

xxvii. URL : http://www.semdo.fr/sempat-2/

xxviii. Pour plus d'information, consulter : https://www.maisondesanteorleans.fr/

xxvix. Décret nº5-603 du 10 juin 1985 relatif à l'hygiène et à la sécurité du travail ainsi qu'à la médecine professionnelle et préventive dans la fonction publique territoriale, art. 2.

xxx. Article L. 162-2 du Code de la sécurité sociale. 
xxxi. Le nom TOPOS pour «Territoires de l'Orléanais, Prospective, Observatoire et Stratégie " reflète l'élargissement du périmètre d'action de l'agence d'urbanisme, anciennement nommée «Agence d'urbanisme de l'agglomération orléanaise ». Désormais, son territoire d'investigation compte, au-delà de la métropole, 4 communautés de communes (Portes de Sologne, Loges, Beauce Loirétaine et Terres du Val de Loire) et 2 PETR (Forêt d'Orléans-Loire-Sologne et Loire Beauce), témoignant ainsi de l'intégration de l'influence de l'agglomération orléanaise sur les territoires périurbains et ruraux qui gravitent autour.

xxxii. Loi du 26 janvier 2016 de modernisation de notre système de santé, dite loi Santé. xxxiii. Cas de la fermeture de la maternité du Blanc (Indre) et de son rattachement à l'hôpital de Châteauroux, au mépris de ses liens naturels avec le CHU de Poitiers. Cas aussi de la situation particulière des franges franciliennes de l'Eure-et-Loir et du Loiret aux liens à renforcer avec Rambouillet, Fontainebleau, Melun, voire des coopérations à établir entre le CHR d'Orléans et le CHU de La Salpêtrière à Paris.

\section{RÉSUMÉS}

L'intervention d'une institution métropolitaine peut-elle remédier à une offre de soins lacunaire pour ses habitants? Cette interrogation guide l'analyse de l'action nouvelle d'Orléans Métropole en matière de santé. Face au nombre insuffisant de médecins pour couvrir les besoins de la population, les maires interviennent par la création de maisons de santé pluridisciplinaire. $\mathrm{Vu}$ l'ampleur des besoins, une réponse strictement communale s'avère néanmoins insuffisante. L'intervention métropolitaine semble légitimée pour coordonner, arbitrer et réguler l'action publique locale. Toutefois, les compétences de la métropole ne sont que partiellement exploitées. En premier lieu, Orléans Métropole a une capacité d'action limitée faute de fondements juridiques explicites pour asseoir sa légitimité et bénéficier d'outils performants. En second lieu, la métropole est à la fois concurrencée à l'échelle extra-métropolitaine par la région et les territoires adjacents, souhaitant échapper à toute satellisation, et à l'échelle intramétropolitaine par les communes membres, souhaitant préserver leur autonomie.

\section{INDEX}

Mots-clés : métropolisation, santé, territoire, lutte d'institutions, gouvernance, intercommunalité

\section{AUTEURS}

\section{PIERRE ALLORANT}

Professeur des universités en histoire du droit, POLEN Université d'Orléans 


\section{SYLVAIN DOURNEL}

Ingénieur de recherche, docteur en géographie et aménagement, CEDETE, Université d'Orléans

FOUAD EDDAZI

Maître de conférences en droit public, CRJ Pothier, Université d'Orléans 